\title{
Blood Pressure and Indices of Glomerular Filtration Area in Hypertensive and Normotensive Prague Rats
}

\author{
H. Hellmann J.M. Davis K. Thurau \\ Physiological Institute, University of Munich, Germany
}

\section{Key Words}

Prague hypertensive rat · Hypertension - Glomerulus numbers and dimensions · Gender differences · Blood pressure

\begin{abstract}
The involvement of the kidney in the pathogenesis of hypertension has long been recognised, although the specific renal mechanisms underlying this phenomenon are still unknown. A current hypothesis attributes hypertension to a reduction in glomerular filtration area by glomerular loss. The present study analyses the relationship between glomerular number and volume and conscious systolic blood pressure (SBP) in 4- to 53-week-old hypertensive (PHR) and normotensive (PNR) rats of the Prague strain. Adult PHRs had higher SBP, were larger and had larger kidneys than PNRs, but $20 \%$ fewer glomeruli. A significant negative correlation between SBP and glomerular number was found in PHR males, but not in PHR females or PNRs. There was no correlation at all between glomerular volume and SBP and, in young animals, both SBP and glomerular number were higher in PHRs than in PNRs. In addition, in adult PHRs, glomerular volume and SBP were higher in males than in females. In summary, a generally valid, causal relation-
\end{abstract}

\begin{tabular}{ll}
\hline KARGER & ( 2000 S. Karger AG, Basel \\
$1420-4096 / 00 / 0236-0385 \$ 17.50 / 0$ \\
Fax +4161306 1234 \\
$\begin{array}{l}\text { E-Mail karger@karger.ch } \\
\text { www.karger.com }\end{array}$ & $\begin{array}{l}\text { Accessible online at: } \\
\text { www.karger.com/journals/kbr }\end{array}$
\end{tabular}

ship linking raised blood pressure to decreased glomerular number or volume could not be demonstrated in the Prague rat model of genetically determined hypertension. The nature of the renal mechanism(s) determining the hypertension in this model remains unknown.

Copyright @ 2000 S. Karger AG, Basel

\section{Introduction}

The role of the kidney in the genesis/maintenance of high blood pressure (BP) in certain models of hypertension has not been more conclusively shown than in the cross-transplantation experiments of Dahl et al. [1], Bianchi et al. [2], Ferrari et al. [3] and others [4, 5]. In these experiments, bilaterally nephrectomised normotensive rats develop hypertension after receiving a kidney transplant from a hypertensive animal, and vice versa. Despite these clear findings, the renal mechanisms leading to hypertension have not been identified. From the frequent association between hypertension and a reduced number of glomeruli [6-9], supported by experimental evidence gained from partial nephrectomy [10], Brenner [8] and Brenner et al. [9] have proposed that the two parameters are linked in a positive feedback, such that a reduced filtration surface area (reduced number of glomeruli and/or

Dr. J.M. Davis

Physiologisches Institut der Universität München Pettenkoferstrasse 12, D-80336 Munich (Germany)

Tel. +49 89 5996550, Fax +49895996532

E-Mailjmd@physiol.med.uni-muenchen.de 
surface area per glomerulus) leads to renal sodium retention and hence to the development of hypertension, which then eventually results in glomerular damage, etc. Attractive as this hypothesis may be, a number of problems are apparent. First, whilst massive nephrectomy $(5 / 6$ or $7 / 8$ of the total renal mass) seems indeed to result regularly in hypertension, reduction in the glomerular number by $50 \%$ (i.e. uninephrectomy) frequently does not $[11,12]$. Moreover, the degree of hypertension seems to be related to the method of nephrectomy [13]. Both these findings are difficult to reconcile with a causal relationship between BP and indices of glomerular filtration area. Should such a relationship exist, one of the principal predictions would be that a correlation exists between the glomerular indices and BP. To our knowledge, such a relationship has not been demonstrated before. The aim of the present study was to assess the BP and glomerular indices in rats of a wide range of ages. The rat strain employed was the Prague strain, for which hypertensive (PHR) and normotensive (PNR) substrains exist [14], and for which the transplantability of the hypertension has been demonstrated [4].

Some of the data from the present study have been presented in a preliminary form $[15,16]$.

\section{Materials and Methods}

\section{Kidney Preparation}

Experiments were carried out on 4- to 53-week-old PNRs and PHRs of both sexes. Animals were bred and held in our own facilities and had access to standard chow (Altomin, Lage, Germany) and tap water ad libitum. On at least 3 separate days prior to the experiment, systolic BP (SBP) was measured by tail-cuff sphygmomanometry. In earlier experiments in the series, the rats were anaesthetised by intraperitoneal injection of $40 \mathrm{mg} / 100 \mathrm{~g}$ body weight pentobarbitone sodium (Nembutal, Abbott), tracheotomised and the left femoral vein cannulated. Alcian blue was then infused to stain the glomeruli in vivo, as described elsewhere [17]. After 20-30 min, the animal was sacrificed by injection of an overdose of the anaesthetic. In later experiments the staining procedure was dispensed with and the animals sacrificed immediately. Glomeruli were counted using a method similar to that described by Damadian et al. [18]. In brief, both kidneys were removed immediately after sacrifice and decapsulated. After weighing, the kidneys were then cut into small pieces and, if stained with Alcian blue, fixed in $1 \% \mathrm{NH}_{4} \mathrm{Cl}$. The kidney fragments were then macerated $(50 \% \mathrm{HCl}$ for $2 \mathrm{~h}$ at room temperature), rinsed and transferred to a flask containing $100 \mathrm{ml} \mathrm{H}_{2} \mathrm{O}$ (or, in later experiments, $0.9 \% \mathrm{NaCl}$, see below). The tissue was then dispersed on a magnetic stirrer at low speed for $8-10 \mathrm{~h}$ and the numbers of glomeruli in $40-\mu 1$ aliquots of the tissue suspension were counted under microscope. There was no difference in the glomerular counts in age-, weight-, gender- and strain-matched animals with or without the Alcian blue stain (data not shown).

\section{Measurements and Calculations}

The mean number $( \pm \mathrm{SD})$ of glomeruli per aliquot for all animals was $21.27 \pm 0.99$. From each preparation new aliquots were taken and counted until the SD was $<5 \%$ of the mean (this required at least 20 aliquots/animal) and the total number of glomeruli for both kidneys calculated. In 5-20 aliquots (i.e. 100-400 glomeruli) glomerular diameter was also measured using a calibrated ocular graticule. The parameters of the size distribution (mean, variance, skewness and kurtosis) were calculated for each animal. Since the mean diameter of glomeruli from kidneys dispersed in distilled water was about $25 \%$ larger than those from kidneys dispersed in $0.9 \% \mathrm{NaCl}$, a correction factor was applied to the measured diameters of the glomeruli dispersed in water. This factor was calculated as the quotient of the mean diameter of glomeruli dispersed in water and that of glomeruli dispersed in $0.9 \% \mathrm{NaCl}$. After application of this correction, the remaining distribution indices (variance, skewness and kurtosis) were not significantly different between the two dispersion fluids (data not shown), and the data were then pooled. The glomerular volume, as a correlate of the filtration area, was calculated from the diameter, assuming spherical geometry and the total glomerular volume estimated as the product of mean glomerular volume and the total number of glomeruli. The total number of glomeruli and the glomerular volume were also normalised with respect to body weight.

\section{Data Analysis}

Data are given as means \pm SD for the number of observations. Although preliminary analysis showed that the formal prerequisites for the use of analysis of variance (Gaussian distribution of variables) and Student's $t$ test were not always met for all variables in all tests, analysis of residuals showed that the deviations from normality were limited and not great. Since ANOVA and the t test are robust with respect to deviations from normality, these tests were employed. As a check, non-parametric tests (Mann-Whitney U test) were also made to test the differences between means. The fact that these tests yielded probability values very similar to ANOVA and $t$ test justified the use of the latter. Partial correlation and regression analyses were used to assess the relationship between $\mathrm{BP}$ and the various glomerular indices. Statistical calculations were made using a commercially available statistics software package (SPSS for Windows v. 8.0, SPSS, Chicago, Ill., USA); graphics were prepared using FigP (v. 2.5, Biosoft, Cambridge, UK). $\mathrm{p}<0.05$ was regarded as significant.

\section{Results}

Figure 1a is a scattergram showing $\mathrm{BP}$ as a function of age. As shown by the lines of best fit, SBP in both PNRs and PHRs increases from the earliest measurable time (about 4 weeks) up to about 8 weeks, and remains relatively constant thereafter. For this reason the animals were divided into young (4- to 7-week-old) and adult (8 weeks and over) groups. ANOVA showed no significant interaction between the fixed variables (gender and strain) so that the genders and strains could be assessed separately. Table 1 summarises the data obtained from all animals. 
Fig. 1. Relationship between age and systolic blood pressure (a) and total number of glomeruli in both kidneys (b) for male and female normotensive (PNR) and hypertensive (PHR) rats of the Prague strain. The lines are the least-squares best fits to curves of the form $y=a / x+b x^{-c}+d$. $\boldsymbol{\bullet},-\quad=$ PNR males; $\mathbf{\square},---=$ PNR females; $\bigcirc,---=$ PHR males; $\square,----=$ PHR females.
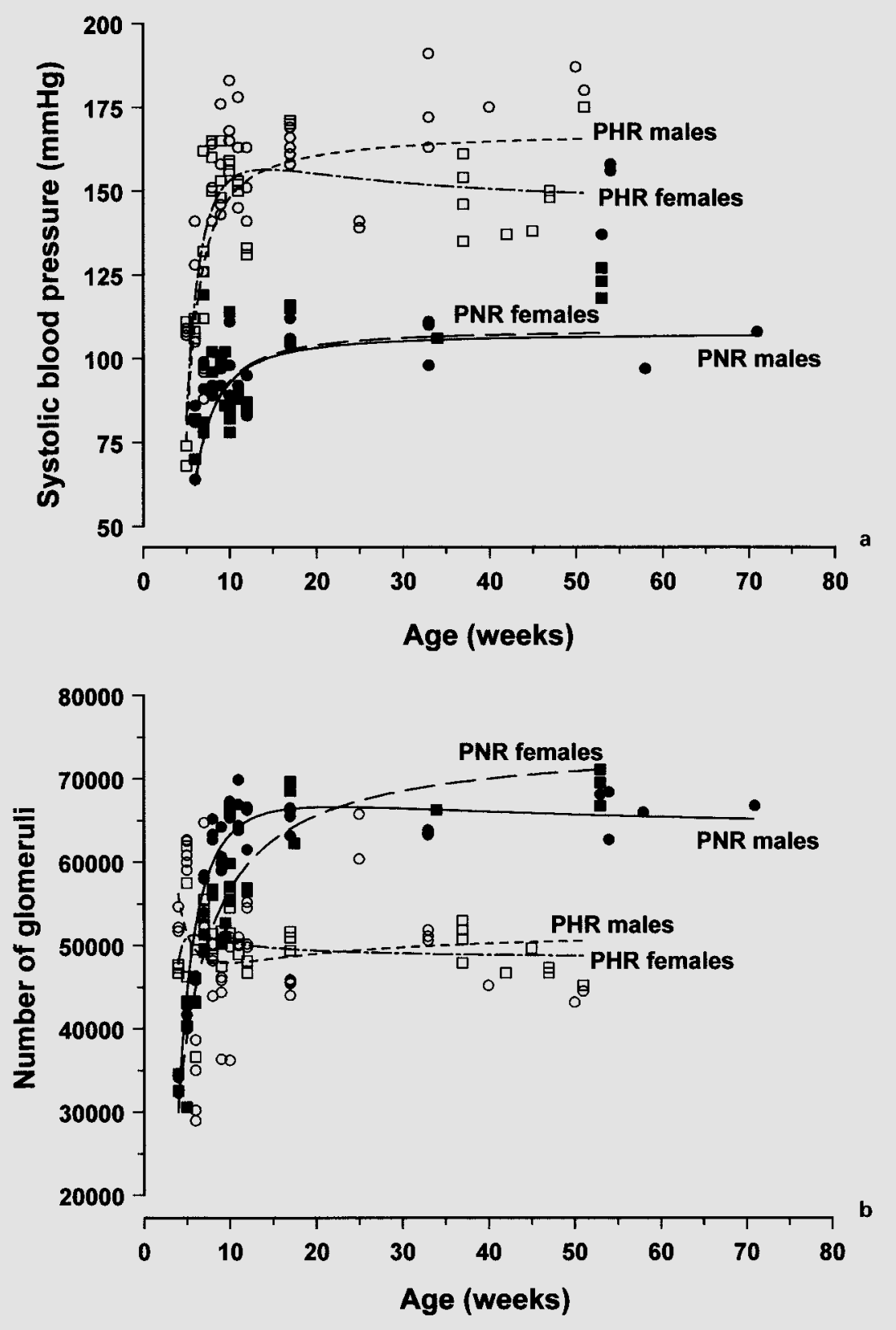

\section{Blood Pressure, Body and Kidney Weights and}

Glomerular Indices

Young Animals. In contrast to the adult animals (see below), the young animals showed no significant gender differences in any of the measured variables. There were, however, substantial differences between the strains. Although not 'hypertensive' in the usual sense (i.e. SBP $>140 \mathrm{~mm} \mathrm{Hg}), \mathrm{SBP}$ in the young PHRs $(112 \pm 21 \mathrm{~mm}$
$\mathrm{Hg}$ ) was already significantly higher than that in PNRs (86 $\pm 15 \mathrm{~mm} \mathrm{Hg}$ ). PHRs had larger kidneys (total kidney weight $1.25 \pm 0.51 \mathrm{~g})$ than PNRs $(0.93 \pm 0.13 \mathrm{~g})$, and the body weights also tended to be greater, although this difference was not significant. Glomerular dimensions (diameter $93 \pm 10$ vs. $92 \pm 11 \mu \mathrm{m}$; volume $4.12 \pm 1.29$ vs. $4.02 \pm 1.42 \mu \mathrm{l} \times 10^{-4}$ for PHRs and PNRs, respectively) were not different, but young PHRs had significantly 
Table 1. Summary of data relating to young (4- to 7-week-old) and adult (over 7-week-old) Prague rats (means \pm SD)

\begin{tabular}{|c|c|c|c|c|c|c|c|c|c|c|c|c|c|}
\hline Group & G & $\mathrm{n}$ & $\begin{array}{l}\text { Age } \\
\text { weeks }\end{array}$ & $\begin{array}{l}\text { BW } \\
\mathrm{g}\end{array}$ & $\begin{array}{l}\text { SBP } \\
\mathrm{mm} \mathrm{Hg}\end{array}$ & $\begin{array}{l}\text { KW } \\
\mathrm{g}\end{array}$ & $\begin{array}{l}\text { KW/BW } \\
\mathrm{g} / 100 \mathrm{~g}\end{array}$ & $\begin{array}{l}\text { Diam } \\
\mu \mathrm{m}\end{array}$ & $\begin{array}{l}\mathrm{v} \\
\mu \mathrm{l} \times 10^{-4}\end{array}$ & $\begin{array}{l}\mathrm{N} \\
\times 10^{3}\end{array}$ & $\begin{array}{l}\text { N/BW } \\
10^{3} / 100 \mathrm{~g}\end{array}$ & $\begin{array}{l}\mathrm{V} \\
\mu \mathrm{l}\end{array}$ & $\begin{array}{l}\mathrm{V} / \mathrm{BW} \\
\mu 1 / 100 \mathrm{~g}\end{array}$ \\
\hline \multicolumn{14}{|l|}{ Young } \\
\hline \multirow[t]{3}{*}{ PNR } & M & $6-12$ & $5.5 \pm 1.2$ & $111 \pm 42$ & $87 \pm 13$ & $1.01 \pm 0.28$ & $0.95 \pm 0.14$ & $93 \pm 11$ & $4.23 \pm 1.57$ & $44.3 \pm 9.1$ & $42.9 \pm 9.5$ & $19.8 \pm 10.6$ & $17.0 \pm 3.5$ \\
\hline & $\mathrm{F}$ & $6-11$ & $5.61 \pm$ & $92 \pm$ & $85 \pm 17$ & $0.85 \pm$ & $0.94 \pm 0.13$ & $90 \pm 11$ & & 41. & 16.0 & & \\
\hline & $\mathrm{M}+\mathrm{F}$ & $12-23$ & $5.6 \pm$ & $102 \pm 40$ & $86 \pm 15$ & $0.93 \pm 0.13$ & $0.94 \pm 0.13$ & $92 \pm 11$ & & 43.2 & $46.4 \pm$ & 18. & 17.3 \\
\hline \multirow[t]{3}{*}{ PHR } & M & $11-16$ & $5.6 \pm 1$ & $139 \pm 69$ & $112 \pm 19^{*}$ & $1.37 \pm 0.58$ & $1.04 \pm 0.13$ & $94 \pm$ & $=1.30$ & $51.6 \pm 11.8$ & $51.8 \pm 33.9$ & $21.5 \pm$ & $18.2 \pm 7.0$ \\
\hline & $\mathrm{F}$ & 27 & $5.6 \pm 1.2$ & $114 \pm 51$ & $111 \pm 27$ & $1.10 \pm 0.38$ & $1.02 \pm 0.14$ & $92 \pm 10$ & $3.96 \pm 1.31$ & $50.3 \pm 6.4^{*}$ & $55.6 \pm 30.3$ & $20.2 \pm 7.5$ & $18.9 \pm 4.2$ \\
\hline & $\mathrm{M}+\mathrm{F}$ & $21-29$ & $5.6 \pm 1.1$ & $128 \pm 61$ & $112 \pm 21^{\dagger}$ & $1.25 \pm 0.51^{\dagger}$ & $1.03 \pm 0.13$ & $93 \pm 10$ & $4.13 \pm 1.29$ & $51.0 \pm 9.6^{\dagger}$ & $53.5 \pm 31.8$ & $20.9 \pm 8.3$ & $18.5 \pm 5.8$ \\
\hline \multicolumn{14}{|l|}{ Adult } \\
\hline \multirow[t]{2}{*}{ PNR } & M & $32-34$ & $22.0 \pm 17.9$ & $296 \pm 51$ & $103 \pm 18$ & $2.02 \pm 0.26$ & $0.69 \pm 0.07$ & $117 \pm 9$ & $8.08 \pm 1.82$ & $64.8 \pm 2.5$ & $22.6 \pm 3.8$ & $=11.4$ & $18.3 \pm 3.2$ \\
\hline & $\mathrm{F}$ & $21-22$ & $18.1 \pm 15.3$ & $186 \pm 31^{\S}$ & $100 \pm 15$ & $1.31 \pm 0.19 \S$ & $0.71 \pm 0.07$ & $111 \pm 9 \S$ & $6.83 \pm 1.68 \S$ & $60.3 \pm 7.0 \S$ & $32.9 \pm 3.6 \S$ & $41.7 \pm 13.8 \S$ & $22.2 \pm 4.4 \S$ \\
\hline \multirow[t]{2}{*}{ PHR } & M & $30-31$ & $18.1 \pm 12.2$ & $342 \pm 64^{*}$ & $161 \pm 15^{*}$ & $2.45 \pm 0.40 *$ & $0.72 \pm 0.09$ & $115 \pm 10$ & $7.88 \pm 1.87$ & $48.4 \pm 6.0^{*}$ & $14.6 \pm 2.8^{*}$ & $38.9 \pm 9.4^{*}$ & $11.5 \pm 2.2 *$ \\
\hline & $\mathrm{F}$ & 27 & $21.5 \pm 15.4$ & $222 \pm 39 * 8$ & $\S 153 \pm 13^{* \S}$ & $1.60 \pm 0.22 * 8$ & $0.73 \pm 0.07$ & $109 \pm 8^{\S}$ & $6.62 \pm 1.42^{\S}$ & $49.8 \pm 2.6^{*}$ & $23.2 \pm 4.4 * \S$ & $\S 32.8 \pm 6.6 * \S$ & $14.9 \pm 2.4 * \S$ \\
\hline
\end{tabular}

$\mathrm{G}=$ Gender; $\mathrm{n}=$ number of animals; $\mathrm{SBP}=$ systolic arterial blood pressure; $\mathrm{BW}=$ body weight; $\mathrm{KW}=$ total weight, both kidneys;

$\mathrm{KW} / \mathrm{BW}=$ kidney weight normalised for body weight; Diam = glomerular diameter; $\mathrm{v}=$ glomerular volume;

$\mathrm{N}=$ total number of glomeruli, both kidneys; N/BW = N normalised for body weight; $\mathrm{V}=$ total glomerular volume;

$\mathrm{V} / \mathrm{BW}=\mathrm{V}$ normalised for body weight $\mathrm{M}=$ male; $\mathrm{F}=$ female; $\mathrm{PNR}=$ normotensive rat $\mathrm{PHR}=$ hypertensive rat.

Two-tailed probability, corrected for non-equal variances: ${ }^{*} \mathrm{p}<0.05$ vs. PNR of same sex; ${ }^{\dagger} \mathrm{p}<0.05$ vs. PNR; ${ }^{\S} \mathrm{p}<0.05$ vs. male of same strain, ANOVA and t test for non-paired samples.

more glomeruli than PNR $\left(51.00 \pm 9.63 \times 10^{3}\right.$ vs. 43.16 $\pm 8.03 \times 10^{3}$, respectively). The mean indices of the parameters describing the form of the distribution of glomerular diameters (skewness and kurtosis) were not significantly different from the values predicted for a normal distribution, nor did they differ between the strains (data not shown). In view of the large scatter, neither the total glomerular volume nor the glomerular indices normalised for body weight were significantly different between the strains.

Adult Animals. Adult PHRs were significantly bigger than adult PNRs, and for each strain the males were significantly larger than the females: $342 \pm 64,222 \pm 39$, $296 \pm 51$ and $186 \pm 31 \mathrm{~g}$ for PHR males and females and PNR males and females, respectively. The gender difference was substantially greater than the strain difference. A similar pattern was seen for kidney weights $(2.45 \pm 0.40$, $1.60 \pm 0.22,2.02 \pm 0.26$ and $1.31 \pm 0.19 \mathrm{~g}$, respectively). These strain and gender differences disappeared when kidney weight was normalised with respect to body weight. Glomerular dimensions differed only between the genders, with males having significantly larger glomerular diameters $(115 \pm 10$ and $117 \pm 9 \mu \mathrm{m}$ for PHRs and PNRs, respectively) than females (109 \pm 8 and $111 \pm$ $9 \mu \mathrm{m}$, respectively), and correspondingly larger glomerular volumes (PHR males and females $7.88 \pm 1.87$ and $6.62 \pm 1.42 \mu 1 \times 10^{-4}$, PNR males and females $8.08 \pm$ 1.82 and $6.83 \pm 1.68 \mu 1 \times 10^{-4}$, respectively). As in the young animals, glomerular diameters were distributed normally. In contrast to the young animals, adult PHRs had significantly fewer glomeruli $(48,390 \pm 5,950$ and $49,780 \pm 2,590$ for males and females, respectively) than adult PNRs $(64,810 \pm 2,500$ and $60,280 \pm 6,960$, respectively). The gender difference and the difference between the young and the adult were significant in PNRs, but not in PHRs. Given the greater body weights of PHRs, normalising the total number of glomeruli with respect to body weight enhanced the interstrain differences, but reversed the gender differences, with females now having proportionately more glomeruli than males: PHR males and females $14,620 \pm 2,810$ and $23,170 \pm 4,350$ per $100 \mathrm{~g}$ body weight, respectively, and PNRs $22,590 \pm$ 3,790 and $32,920 \pm 3,630$ per $100 \mathrm{~g}$ body weight, respectively. A similar pattern was seen for total glomerular volume, which was significantly larger in PNRs than in PHRs and in males than in females. Normalisation with respect to body weight increased the relative strain difference, and again reversed the gender difference (table 1).

\section{Relationship between Blood Pressure and Glomerular Indices}

The relationship between SBP and the glomerular indices is analysed in figure 2 . Since the ranges over which BP and the glomerular indices varied were relatively small and were different between the gender race groups, the correlation analysis is difficult, and is addressed only 
Fig. 2. Relationship between systolic blood pressure and the total number of glomeruli in both kidneys $(\mathrm{N})(\mathbf{a})$ and the total glomerular volume $(\mathrm{V})(\mathbf{b})$. The lines show the corresponding bivariate linear regressions for the two strains and genders. Lines and symbols as in figure 1 .
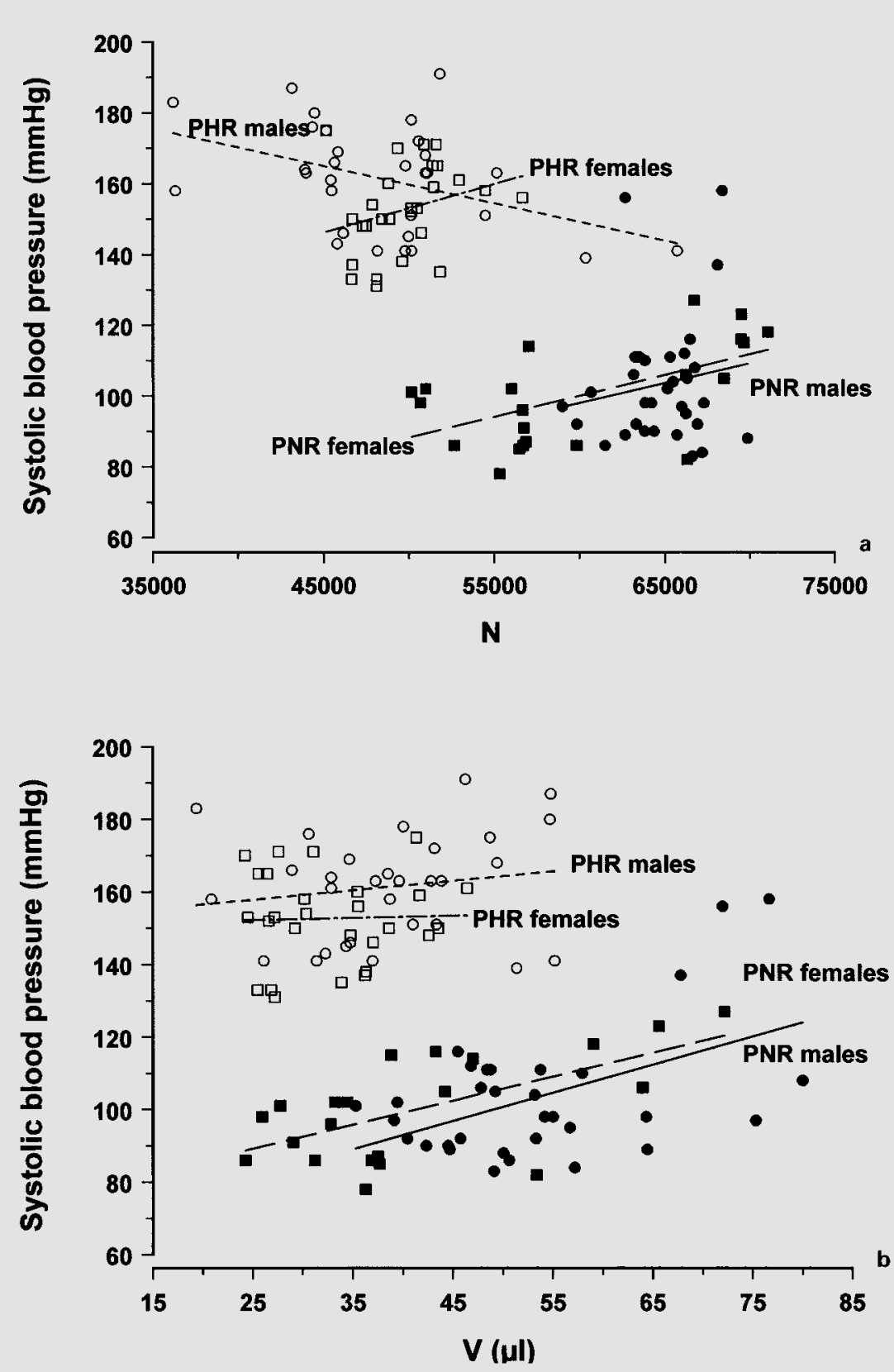

qualitatively. There were significant (one-sided) bivariate correlations between BP and the normalised glomerular indices in the young animals, and for both absolute and normalised indices in the adult animals. To establish the source of this significance, variables were eliminated progressively. Eliminating the time-dependent variables (age, body and kidney weight) abolished all significance in the young animals, so that the analysis in these animals was

Hypertension and Glomerular Number in Prague Rats not pursued further. In the adult animals, eliminating the time-dependent variables had no effect on the significance of the correlations, nor did eliminating the effect of gender. Eliminating the effect of strain, however, completely abolished all significance in these animals. To establish the source of the strain-dependent significance, the adults were then subdivided on the basis of strain. Although the correlation analysis implied practically no

Kidney Blood Press Res 2000;23:385-392 389 
effect of gender, in view of the gender-related differences in table 1, further subdivision according to gender was deemed reasonable. The further analysis is shown in figure 2. Figure 2 shows scatter plots of SBP as a function of glomerular number (fig. 2a) or total glomerular volume (fig. 2b) for the four strain/gender combinations. It is apparent that only the PHR males show a negative correlation between BP and glomerular number, whilst none of the groups showed a negative correlation with glomerular volume. Indeed, as the simple regression lines (the solid lines in figure 2) show, the slopes were frequently positive. The positive correlations between BP and total glomerular volume for PNR males and females and glomerular number for PNR females were significant. This significance is lost, however, when the effects of the time-dependent variables are removed (partial regression analysis). After this procedure, the only significant correlation (of either sign) remaining is the negative relationship between BP and glomerular number for PHR males.

\section{Discussion}

The questions addressed in this study were: are there systematic differences in the glomerular indices between PNR and PHR, and, if so, is there a correlation between $\mathrm{BP}$ and all or any of these indices. There first question can be answered with yes. Although glomerular dimensions did not differ between the strains, PHRs had about 20\% fewer glomeruli than PNRs and a correspondingly smaller total glomerular volume. These findings agree in this respect with one study in the spontaneously hypertensive rat (SHR) [19], but not with others [20, 21]. The second question cannot be answered unequivocally, as will be seen below. Before going into this matter, the methods employed in the study will be addressed briefly.

\section{Critique of Methods}

Since the tail-cuff method cannot be used to measure BP in rats until the tails have an adequate size (about 4 weeks in the Prague rats) and glomeruli cannot be counted accurately using the maceration method in the early postnatal period (days P1-P14) in which the Sshaped bodies could be mistaken for glomeruli and the statistical error per aliquot is too high, it was not possible to examine in detail the relationship between SBP and the glomerular indices until about 4 weeks after birth.

The range of glomerular numbers and dimensions observed in this study agree quite well with published values (25,000-32,000 glomeruli/kidney, diameter 92-
$117 \mu \mathrm{m}$, volume $4-8 \mu \mathrm{l} \times 10^{-4}$, total glomerular volume $16-50 \mu$ l) [19-22]. It must be noted that total glomerular volume is a semiquantitative estimate at the best, in view of the accuracy with which the ocular graticule can be read and the often non-spherical glomerular geometry. Recognisable glomerular fragments were observed only very rarely, so that mechanical disruption of glomeruli, leading to a significant underestimation of numbers, is unlikely.

Table 1 and figure $1 \mathrm{~b}$ show a significant increase in glomerular number between young and adult PNRs (but not PHRs). Although analogous findings have been reported elsewhere [23-25], but not consistently [22, 26], this is difficult to account for if, as is now generally assumed [27], nephrogenesis in the rat is complete by $1-2$ weeks of age. An explanation for this discrepancy based on the methods used to isolate and count the glomeruli would require that the number of glomeruli be systematically underestimated specifically in young PNRs, but not PHRs. Whilst it is feasible, for instance, that a putative underestimation may be related to the higher glomerular density (number per gram kidney weight) in young PNRs, analysis of this parameter for the strains, ages and genders (table 1) fails to reveal any consistent pattern. Thus, although a methodological explanation cannot be unequivocally excluded, this seems rather unlikely. The following argument may account for this phenomenon. Although the study was not designed as a study of growth and development in the Prague strain, analysis of the data implies a growth abnormality, particularly affecting PNR. A comparison of body weights with those of standard outand inbred stains [28] shows that at 4 weeks, PNRs weigh about $30 \%$ less than outbred rat strains such as Wistar or Sprague-Dawley, but are comparable with inbred strains such as Wistar-Kyoto (WKY), Brown-Norway or Lewis. PHRs at this age are even less well developed, weighing about $25 \%$ less than PNRs. At 5 weeks and later, the pattern is different: PNR males now weigh about $30-40 \%$ less than the males of outbred strains and $20-30 \%$ less than males of inbred stains, whilst PHR weights are roughly equivalent to those of inbred strains and hence are now substantially larger than PHRs. This is the situation reflected by the means in table 1 , since there were more 6-and 7-week-old rats in the group of young animals in the present study than 4-week-old rats. This growth pattern is consistent with an inbreeding-induced growth retardation, affecting mainly PNRs. Since the rate of growth is presumably genetically determined, a corollary might be delayed cessation of nephrogenesis, thus accounting for the relatively low numbers of glomeruli in the youngest PNRs and depressing the mean value in this 
group. It is emphasised that this argument is entirely speculative. In the final analysis, this and the earlier findings [23-25] remain unexplained.

\section{Relationship between BP and the Glomerular Indices}

As noted above, PHRs have higher BP but fewer glomeruli than PNRs. Indeed, bivariate correlation calculations reveal significant negative correlations between SBP and some (young animals) or all (adult animals) of the glomerular indices. At first glance this would appear to be consistent with a causal relationship between the glomerular indices and BP in the sense suggested by Brenner et al. [9]. However, partial correlation and regression analyses show that this conclusion must be revised. In the young animals, the correlation is lost when the effects of the three time-dependent variables (age and body and kidney weights) are removed. This implies, not unexpectedly, that BP in young animals increases with age (fig. 1a), but that this is also independent of the glomerular indices. The further analysis in adult animals shows that the significant bivariate correlations are still present after controlling for the time-dependent variables and additionally for sex, but is lost totally when controlling additionally for strain or sex and strain. On the basis of these results, the adult animals were divided according to strain and sex, and regression of SBP on the glomerular indices calculated (fig. 2). The simple regression lines in figure 2 show that the only negative correlation between a glomerular index and SBP is with glomerular number in the PHR males. The remaining correlations are either not significant or, if significant, positive. In the latter case the significance is lost after removing the effects of the timedependent variables. In short, detailed data analysis indicates that the significant correlation between glomerular number and SBP seen in the entire sample is due solely to the PHR males. There is no correlation between total glomerular volume and BP in any of the subgroups.

There are, in fact, numerous arguments against a generally valid, causal relationship between SBP and indices of glomerular filtration area (glomerular number or total glomerular volume, raw or normalised). First, if such a hypothesis is to be valid generally, SBP would be expected to correlate with glomerular number and total glomerular volume in all strain/gender pairs. As indicated above, a correlation with glomerular number was observed only in 1 of the 4 groups (PHR males) and there was no correlation with total glomerular volume in any group, although given the uncertainty and scatter of total glomerular volume this argument carries less weight. Second, the slope of the partial regression between SBP and glomerular

Hypertension and Glomerular Number in Prague Rats number in PHR males means that increasing glomerular number from 48,000 (mean of the PHR males) up to 65,000 (mean in male PNRs) would reduce SBP by only $19 \mathrm{~mm} \mathrm{Hg}$, i.e. only one third of the observed difference in SBP. In other words, even though the correlation is significant, the effect is small. Third, SBP is significantly higher already in young PHRs vs. PNRs [14], but glomerular number is also significantly higher in PHRs than in PNRs (the other indices show the same trend but without achieving significance). A comparable argument may be drawn from the following observation: when kidneys from genetically hypertensive donor rats kept normotensive by antihypertensive drug therapy are transplanted into bilaterally nephrectomised normotensive recipients, the latter still develop hypertension [4, 5, 29]. This shows that the defect in the kidney causing hypertension is independent of BP. Fourth, within the PHRs, males have significantly larger glomeruli than females but glomerular number is not significantly different, so that total glomerular volume is significantly larger in the males than in the females. However, SBP is also significantly higher in the males. Fifth, as noted in the preliminary reports of some of these data [15, 16], subsets of PHRs and PNRs with same SBP (selected where the distributions overlap) have different glomerular counts; PHR and PNR subsets with same glomerular counts have different SBPs, i.e. the strain-dependent difference in one variable is independent of the other. Finally, there is considerable inconsistency in the literature, with some studies reporting no difference in glomerular number/dimensions between SHRs and its control strain WKY [21] or between the fawnhooded rat and its control strain Wistar Albino Glaxo [30]. The final link in the positive feedback envisaged by Brenner et al. [9] and Brenner and Anderson [31] is the induction of glomerular damage by a raised hydrostatic pressure in the glomerular capillaries. Whilst there is no information available on glomerular capillary pressures in the Prague rats, the findings in other hypertensive strains are quite variable. In SHR, for instance, glomerular capillary pressure may be higher, at least in older animals [32], or lower [33], or not different [34] from normotensive controls. For the Milan strain both raised [35] and unchanged [36] glomerular capillary pressures have been reported.

In summary, a causal relationship linking raised BP to decreased glomerular number/volume could not be demonstrated in the Prague rat model of genetically determined hypertension. Although the possibilities of the pattern being established in the period for which there are no data (up to 4 weeks), or of a sex linkage affecting males,

Kidney Blood Press Res 2000;23:385-392 391 
cannot be excluded, the nature of the genetic defect in the kidney which determines the hypertension remains unknown at the present. The findings also emphasise the pitfalls involved in extrapolating from one specific model of kidney-related hypertension (here renal ablation) to all models.

\section{Acknowledgements}

The authors gratefully acknowledge the skilled assistance of Mrs. Gisela Schubert, the fruitful discussions with Prof. M. Horster, and the assistance of Dr. M. Wiseman, Leibniz Rechenzentrum, Munich, with SPSS.

\section{References}

1 Dahl LK, Heine M, Thompson K: Genetic influence of the kidneys on blood pressure. Evidence from chronic renal homografts in rats with opposite predispositions to hypertension. Circ Res 1973;34:94-101.

2 Bianchi G, Fox U, Di Francesco GF, Giovanetti AM, Pagetti D: Blood pressure changes produced by kidney cross-transplantation between spontaneously hypertensive rats and normotensive rats. Clin Sci Mol Med 1974;47:435448.

3 Ferrari P, Barber BR, Torielli L, Ferrandi M, Salardi S, Bianchi G: The Milan hypertensive rat as a model for studying cation transport abnormality in genetic hypertension. Hypertension 1987;10:I32-I36.

4 Heller J, Schubert G, Havlickova J, Thurau K: The role of the kidney in the development of hypertension - A transplantation study in the Prague hypertensive rat. Pflügers Arch 1993; 425:208-212.

5 Kopf D, Waldherr R, Rettig R: Source of kidney determines blood pressure in young renal transplanted rats. Am J Physiol 1993;265: F104-F111.

6 Hakim RM, Goldszer RC, Brenner BM: Hypertension and proteinuria: Long-term sequelae of uninephrectomy in humans. Kidney Int 1984;25:930-936.

7 Langley-Evans SC, Welham SJ, Jackson AA: Fetal exposure to a maternal low protein diet impairs nephrogenesis and promotes hypertension in the rat. Life Sci 1999;64:965-974.

8 Brenner BM: Nephron adaptation to renal injury or ablation. Am J Physiol 1985;249:F324F337.

9 Brenner BM, Garcia DL, Anderson S: Glomeruli and blood pressure. Less of one, more of the other? Am J Hypertens 1988;1:335-347.

10 Anderson S, Meyer TW, Rennke HG, Brenner BM: Control of glomerular hypertension limits glomerular injury in rats with reduced renal mass. J Clin Invest 1985; 76:612-619.

11 Miller PL, Rennke HG, Meyer TW: Glomerular hypertrophy accelerates hypertensive glomerular injury in rats. Am J Physiol 1991;261: F459-F465.

12 Brown L, Duce B, Miric G, Sernia C: Reversal of cardiac fibrosis in deoxycorticosterone acetate-salt hypertensive rats by inhibition of the renin-angiotensin system. J Am Soc Nephrol 1999;10(suppl 11):143-148.
13 Ibrahim HN, Hostetter TH: The renin-aldosterone axis in two models of reduced renal mass in the rat. J Am Soc Nephrol 1998;9:72-76.

14 Heller J, Hellerova S, Dobesova Z, Kunes J, Zicha J: The Prague hypertensive rat: A new model of genetic hypertension. Clin Exp Hypertens 1993;15:807-818.

15 Hellmann H, Davis JM, Thurau K: Glomerulus number and blood pressure in genetically identical hypertensive and normotensive (Prague) rats (abstract P837). Proc 14th Int Cong Nephrol 1997, p 283.

16 Hellmann H, Davis JM, Thurau K: Glomerulus number and blood pressure in the Prague hypertensive rat. Kidney Int 1998;54(suppl 67):211-212.

17 Bankir L, Hollenberg NK: In vivo staining of the kidney with Alcian blue: An adjunct to morphological and physiological studies. Renal Physiol 1983;6:151-155.

18 Damadian RV, Shawayri E, Bricker NS: On the existence of non-urine forming nephrons in the diseased kidney of the dog. J Lab Clin Med 1965;65:26-39.

19 Skov K, Nyengaard JR, Korsgaard N, Mulvany MJ: Number and size of renal glomeruli in spontaneously hypertensive rats. J Hypertens 1994;12:1373-1376.

20 Kett MM, Alcorn D, Bertram JF, Anderson WP: Glomerular dimensions in spontaneously hypertensive rats: Effects of AT1 antagonism. J Hypertens 1996;14:107-113.

21 Kett MM, Anderson WP, Bertram JF, Alcorn $\mathrm{D}$ : Structural changes in the renal vasculature in the spontaneously hypertensive rat: No effect of angiotensin II blockade. Clin Exp Pharmacol Physiol Suppl 1996;3:132-135.

22 Nyengaard JR: Number and dimensions of rat glomerular capillaries in normal development and after nephrectomy. Kidney Int 1993;43: 1049-1057.

23 Bonvalet JP, Champion M, Wanstok F, Berjal $\mathrm{G}$ : Compensatory renal hypertrophy in young rats: Increase in the number of nephrons. Kidney Int 1972;1:391-396.
24 Kunes J, Karen P, Capek K, Jelinek J: The influence of salt intake on glomerular count in compensatory kidney hypertrophy in rats of different ages. Experientia 1976;32:887-888.

25 Canter CE, Goss RJ: Induction of extra nephrons in unilaterally nephrectomized immature rats. Proc Soc Exp Biol Med 1975;188:294296.

26 Kaufman JM, Hardy R, Hayslett JP: Agedependent characteristics of compensatory renal growth. Kidney Int 1975;8:21-26.

27 Kazimierczak J: Morphology and enzyme histochemistry of the kidney during normal and compensatory growth in immature rats; in Spitzer A (ed): The Kidney during Development. Morphology and Function. New York, Masson, 1982, pp 31-38.

28 Charles River Deutschland: Research Models. Sulzfeld, Charles River Deutschland, 1999.

29 Rettig R, Folberth C, Stauss H, Kopf D, Waldherr R, Unger T: Role of the kidney in primary hypertension: A renal transplantation study in rats. Am J Physiol 1990;258:F606-F611.

30 de Keijzer MH, Provoost AP, Molenaar JC: Glomerular hyperfiltration in hypertensive fawn-hooded rats. Renal Physiol Biochem 1988;11:103-108.

31 Brenner BM, Anderson S: The interrelationships among filtration surface area, blood pressure, and chronic renal disease. J Cardiovasc Pharmacol 1992;19(suppl 6):1-7.

32 Iversen BM, Amann K, Kwam FI, Wang X, Ofstad J: Increased glomerular capillary pressure and size mediate glomerulosclerosis in SHR juxtamedullary cortex. Am J Physiol 1998;274:F365-F373.

33 Feld LG, Zamlauski-Tucker MJ, Springate JE, Van Liew JB: Single nephron hemodynamics in spontaneously hypertensive rats. Proc Soc Exp Biol Med 1995;209:185-189.

34 Dilley JR, Stier CT Jr, Arendshorst WJ: Abnormalities in glomerular function in rats developing spontaneous hypertension. Am J Physiol 1984;246:F12-F20.

35 Baer PG, Bianchi G: Renal micropuncture study of normotensive and Milan hypertensive rats before and after development of hypertension. Kidney Int 1978;13:452-466.

36 Boberg U, Persson AEG: Increased tubuloglomerular feedback activity in Milan hypertensive rats. Am J Physiol 1986;250:F967F974. 